BUHEP-01-14

\title{
Light Quark Masses with Overlap Fermions in Quenched QCD
}

\author{
L. Giusti, C. Hoelbling, C. Rebbi \\ Department of Physics - Boston University \\ 590 Commonwealth Avenue, Boston MA 02215, USA
}

\begin{abstract}
We present the results of a computation of the sum of the strange and average up-down quark masses with overlap fermions in the quenched approximation. Since the overlap regularization preserves chiral symmetry at finite cutoff and volume, no additive quark mass renormalization is required and the results are $\mathcal{O}(a)$ improved. Our simulations are performed at $\beta=6.0$ and volume $V=16^{3} \times 32$, which correspond to a lattice cutoff of $\sim 2 \mathrm{GeV}$ and to an extension of $\sim 1.4 \mathrm{fm}$. The logarithmically divergent renormalization constant has been computed non-perturbatively in the RI/MOM scheme. By using the $K$-meson mass as experimental input, we obtain $\left(m_{s}+\hat{m}\right)^{\mathrm{RI}}(2 \mathrm{GeV})=120(7)(21) \mathrm{MeV}$, which corresponds to $m_{s}^{\overline{\mathrm{MS}}}(2 \mathrm{GeV})=$ $102(6)(18) \mathrm{MeV}$ if continuum perturbation theory and $\chi \mathrm{PT}$ are used. By using the GMOR relation we also obtain $\langle\bar{\psi} \psi\rangle^{\overline{\mathrm{MS}}}(2 \mathrm{GeV}) / N_{f}=-0.0190(11)(33) \mathrm{GeV}^{3}=-[267(5)(15) \mathrm{MeV}]^{3}$.
\end{abstract}




\section{Introduction}

Quark masses are fundamental parameters of the Standard Model which cannot be measured directly by experiment, since quarks are confined into hadrons. If defined as effective couplings in the Lagrangian, their values can be determined by comparing a theoretical calculation of a given physical quantity (sensitive to quark masses) with the corresponding experimental value. As a consequence quark masses depend on the renormalization scheme and scale, as well as on the fundamental action.

At present the most precise values of light-quark mass ratios (which are scheme and scale independent for mass independent renormalization schemes) are extracted by comparing $K$ - and $\pi$ meson mass ratios with predictions from chiral perturbation theory $(\chi \mathrm{PT})$ [1]. A detailed analysis gives [2]

$$
\frac{m_{u}}{m_{d}}=0.553 \pm 0.043, \quad \frac{m_{s}}{\hat{m}}=24.4 \pm 1.5
$$

where $\hat{m}=\left(m_{u}+m_{d}\right) / 2$. The absolute scale cannot be fixed by $\chi \mathrm{PT}$. It can be determined by comparing non-perturbative lattice QCD computations [3]-[13] or phenomenological estimates [14, 15] with experimental results.

In the last few years much effort has been devoted within the lattice community to obtain a precise determination of the light quark masses in the quenched approximation. Major improvements came with the use of non-perturbative (NP) renormalization techniques for renormalizing the bare quark masses and with the implementation of $\mathcal{O}(a)$ improved actions and operators [6, 8, 9, 12] (for a recent review see [16]). First results from unquenched simulations have also been reported [7, 13, 16].

In this paper we present the results of the first fully non-perturbative computation of $\left(m_{s}+\hat{m}\right)$ with overlap fermions. In this fermionic regularization, flavor and chiral symmetries are preserved at finite lattice spacing and finite volume. As a consequence no additive quark mass renormalization is required and no parameters have to be fine tuned in order to compute $\mathcal{O}(a)$ improved masses and matrix elements. To avoid uncertainties due to lattice perturbation theory, we compute the logarithmic divergent renormalization constant non-perturbatively in the RI/MOM scheme [17. By comparing the experimental $K$-meson mass with the value obtained in our simulations and after a careful analysis of the systematic uncertainties we find as our main result

$$
\left(m_{s}+\hat{m}\right)^{\mathrm{RI}}(2 \mathrm{GeV})=120 \pm 7 \pm 21 \mathrm{MeV}
$$

This value corresponds to

$$
m_{s}^{\overline{\mathrm{MS}}}(2 \mathrm{GeV})=102 \pm 6 \pm 18 \mathrm{MeV}
$$

if next-to-next-to-leading order $\left(\mathrm{N}^{2} \mathrm{LO}\right)$ continuum perturbative results and Eq. (何) are used.

We also report results for the chiral condensate $\langle\bar{\psi} \psi\rangle$ which we compute from the Gell-Mann, Oakes and Renner (GMOR) relation. Our best determination is

$$
\frac{1}{N_{f}}\langle\bar{\psi} \psi\rangle^{\mathrm{RI}}(2 \mathrm{GeV})=-0.0167 \pm 0.0010 \pm 0.0029 \mathrm{GeV}^{3}
$$


which corresponds to

$$
\frac{1}{N_{f}}\langle\bar{\psi} \psi\rangle^{\overline{\mathrm{MS}}}(2 \mathrm{GeV})=-0.0190 \pm 0.0011 \pm 0.0033 \mathrm{GeV}^{3}=-[267 \pm 5 \pm 15 \mathrm{MeV}]^{3}
$$

We have also computed the chiral condensate directly. Even if it requires a severe chiral extrapolation, the direct determination gives results consistent with Eqs. (四), (5).

The first error in Eqs. (2)-(5) represents the statistical error, obtained with the jackknife method. The second error represents our estimate of systematic effects. It should be noted that, while the systematic error due to quenching alone (defined as the quenching error in the limit of zero lattice spacing and infinite volume) should be the same in all fermionic regularizations, discretization errors do depend on the fermionic lattice action and are likely to be smaller in the overlap formulation, because of its good chirality properties. The very good agreement of our results with the current lattice world averages [16] provides further confirmation that the overlap formulation is a suitable regularization for large-scale phenomenological computations.

This paper is organized as follows: in Sec. 2 we set our notation and define the renormalized quark masses and chiral condensate in the overlap regularization; in Sec. 3 we give details about the simulation and some of the results for the meson masses and matrix elements; in Sec. A we present our main results and discuss their systematic errors; Sec. 5 is devoted to our concluding remarks.

\section{Quark Masses and Chiral Condensate with the Overlap Action}

The QCD lattice action in the overlap regularization reads [18]

$$
S=\frac{6}{g_{0}^{2}} \sum_{P}\left[1-\frac{1}{6} \operatorname{Tr}\left[U_{P}+U_{P}^{\dagger}\right]\right]+\bar{\psi}\left[\left(1-\frac{1}{2 \rho} a M\right) D+M\right] \psi
$$

where, in standard notation, $U_{P}$ is the Wilson plaquette, $g_{0}=\sqrt{6 / \beta}$ is the bare coupling constant, $\psi$ and $\bar{\psi}$ carry implicit color, spin and flavor indices, and $M$ is a diagonal matrix of bare masses $\left(m_{1}, m_{2}, \ldots\right)$ in flavor space. $D$ is the Neuberger-Dirac operator defined as

$$
\begin{aligned}
D & =\frac{\rho}{a}(1+V)=\frac{\rho}{a}\left(1+X \frac{1}{\sqrt{X^{\dagger} X}}\right) \\
X & =D_{W}-\frac{1}{a} \rho
\end{aligned}
$$

where

$$
D_{W}=\frac{1}{2} \gamma_{\mu}\left(\nabla_{\mu}+\nabla_{\mu}^{*}\right)-\frac{r}{2} a \nabla_{\mu}^{*} \nabla_{\mu}
$$

is the Wilson-Dirac operator, $0<r \leq 1$ and $0<\rho<2 r$. In our calculations we used $r=1$. $\nabla_{\mu}$ and $\nabla_{\mu}^{*}$ in Eq. (8) are the forward and backward lattice covariant derivatives, defined by

$$
\begin{aligned}
\nabla_{\mu} \psi(x) & =\frac{1}{a}\left[U_{\mu}(x) \psi(x+a \hat{\mu})-\psi(x)\right] \\
\nabla_{\mu}^{*} \psi(x) & =\frac{1}{a}\left[\psi(x)-U_{\mu}^{\dagger}(x-a \hat{\mu}) \psi(x-a \hat{\mu})\right]
\end{aligned}
$$


where $U_{\mu}(x)$ are the lattice gauge links. The fermionic operator of the overlap action satisfies the Ginsparg-Wilson relation 19

$$
\gamma_{5} D+D \gamma_{5}=\frac{a}{\rho} D \gamma_{5} D
$$

which implies an exact continuous symmetry of the action in the massless limit [20]. This symmetry may be interpreted as a lattice form of chiral invariance at finite cutoff

$$
\delta \psi=\hat{\gamma}_{5} \psi, \quad \delta \bar{\psi}=\bar{\psi} \gamma_{5}
$$

where $\hat{\gamma}_{5}$ is defined as

$$
\hat{\gamma}_{5}=\gamma_{5}\left(1-\frac{a}{\rho} D\right)
$$

and satisfies

$$
\hat{\gamma}_{5}^{\dagger}=\hat{\gamma}_{5}, \quad \hat{\gamma}_{5}^{2}=1
$$

The anomaly is recovered from the variation of the measure under the rotations in Eq. (12) [20, 21].

The invariance of the action under non-singlet chiral transformations, defined including a flavor group generator in Eq. (12), forbids mixing among operators of different chirality [22] and therefore:

- No additive quark mass renormalization is required. The quark mass which enters the vector and axial Ward identities is the bare parameter $m(a)$ in the action of Eq. (6).

- Masses and matrix elements are affected only by $\mathcal{O}\left(a^{2}\right)$ discretization errors. No fine tuned parameters are required to remove $\mathcal{O}(a)$ effects.

- The chiral condensate does not require subtractions of power divergent terms (in the chiral limit).

The renormalized quark mass is defined as

$$
\bar{m}(\mu)=\lim _{a \rightarrow 0} Z_{m}(a \mu) m(a)
$$

where $Z_{m}(a \mu)$ is a logarithmically divergent renormalization constant which has to be fixed, for a given scale $\mu$, in a given renormalization scheme. It is worth noting that even if $m(a)$ is the bare parameter which enters the fundamental action in Eq. (6), its relation to a given experimental result is fixed by a non-perturbative lattice QCD calculation and therefore its value is determined up to $\mathcal{O}\left(a^{2}\right)$ terms only.

The bare chiral condensate is defined as

$$
\chi(a) \equiv \lim _{m \rightarrow 0} \frac{1}{N_{f}}\left\langle\bar{\psi}(0)\left[\left(1-\frac{a}{2 \rho} D\right) \psi\right](0)\right\rangle
$$

where $m$ is a common mass given to the light quarks. It satisfies the integrated non-singlet chiral Ward identity

$$
\frac{1}{N_{f}}\left\langle\bar{\psi}(0)\left[\left(1-\frac{a}{2 \rho} D\right) \psi\right](0)\right\rangle=m \sum_{x}\left\langle P(x) P^{c}(0)\right\rangle
$$


where

$$
\begin{aligned}
P(x) & =\bar{\psi}_{1}(x) \gamma_{5}\left[\left(1-\frac{a}{2 \rho} D\right) \psi_{2}\right](x) \\
P^{c}(x) & =\bar{\psi}_{2}(x) \gamma_{5}\left[\left(1-\frac{a}{2 \rho} D\right) \psi_{1}\right](x)
\end{aligned}
$$

correspond the non-singlet pseudoscalar density with degenerate quarks $\left(m_{1}=m_{2}\right)$ and its conjugate. For non-zero mass the chiral condensate is still divergent and it behaves as

$$
\frac{1}{N_{f}}\left\langle\bar{\psi}(0)\left[\left(1-\frac{a}{2 \rho} D\right) \psi\right](0)\right\rangle=\chi(a)+\beta_{\chi} \frac{m(a)}{a^{2}}
$$

where we have taken into account that chiral symmetry forces the coefficient of the linear divergence to be zero.

By writing the correlation function $\left\langle P(x) P^{c}(0)\right\rangle$ as a time-ordered product and by inserting a complete set of states in standard fashion we can also write

$$
\chi(a)=-\lim _{m \rightarrow 0} \frac{m}{M_{P}^{2}}|\langle 0|P| P\rangle|^{2},
$$

where $M_{P}$ is the mass of the pseudoscalar state $|P\rangle$. If we use

$$
2 m|\langle 0|P| \pi\rangle|=f_{P} M_{P}^{2}
$$

where $f_{P}$ is the corresponding pseudoscalar decay constant, we arrive to the familiar GMOR relation

$$
\chi(a)=-\lim _{m \rightarrow 0} \frac{f_{P}^{2} M_{P}^{2}}{4 m}
$$

The renormalized chiral condensate is defined as

$$
\frac{1}{N_{f}}\langle\bar{\psi} \psi\rangle(\mu)=\lim _{a \rightarrow 0} Z_{S}(a \mu) \chi(a)
$$

where $Z_{S}(\mu a)$ can be chosen to be the renormalization constant of the corresponding non-singlet scalar density which, thanks to the flavor symmetry, satisfies $Z_{S}(\mu a)=1 / Z_{m}(\mu a)$. In principle $Z_{S}(\mu a)$ can be computed in perturbation theory [23, 24], but uncertainties due to higher order terms can be avoided using non-perturbative renormalization procedures [17, 25, 11]. The implementation of the RI/MOM technique [17] is straightforward in the overlap regularization, and it allows one to compute $\mathcal{O}(a)$ improved renormalization constants for generic composite operators. In the following we will use the numerical value of $Z_{S}^{\mathrm{RI}}(\mu a)$ we have obtained in Ref. [26] (for an alternative approach see Ref. [27]). 


\section{Numerical Details}

We performed our simulation in quenched QCD with $\beta=6.0$ and $V=16^{3} \times 32$. We used a sample of 54 gauge configurations, generated with the standard Wilson gluonic action of Eq. (6), which we retrieved from the repository at the "Gauge Connection" [28]. We computed overlap propagators from a local source for bare quark masses $m a=0.040,0.055,0.070,0.085,0.100$ and $\rho=1.4$ (and, as already mentioned, $r=1.0$ ). For the calculation of the propagators, we used an optimal rational approximation to the sign of the Hermitian Wilson operator, as proposed in Ref. [29, 30], after explicit evaluation of the contributions from the lowest eigenvectors of $X^{\dagger} X$, and nested multi-conjugate gradient inversions. Details of the numerical implementation will be presented in Ref. [26]. From the propagators, we computed in the standard manner the two-points correlation functions

$$
\begin{aligned}
G_{S S}(t) & =\sum_{x}\left\langle S(x, t) S^{c}(0,0)\right\rangle \\
G_{P P}(t) & =\sum_{x}\left\langle P(x, t) P^{c}(0,0)\right\rangle \\
G_{\nabla A P}(t) & =\sum_{x}\left\langle\bar{\nabla}_{0} A_{0}(x, t) P^{c}(0,0)\right\rangle
\end{aligned}
$$

where $P(x, t)$ and $P^{c}(x, t)$ have been defined in Eqs. (18) and (19),

$$
\begin{aligned}
S(x) & =\bar{\psi}_{1}(x)\left[\left(1-\frac{a}{2 \rho} D\right) \psi_{2}\right](x) \\
A_{\mu}(x) & =\bar{\psi}_{1}(x) \gamma_{\mu} \gamma_{5}\left[\left(1-\frac{a}{2 \rho} D\right) \psi_{2}\right](x)
\end{aligned}
$$

$S^{c}(x)$ is defined analogously to Eq. (19) and $\bar{\nabla}_{0}$ denotes the symmetric lattice derivative in the time direction. To improve statistics $G_{S S}(t)$ and $G_{P P}(t)$ have been symmetrized around $t=T / 2$ $\left(T=N_{t}=32\right.$ ). We estimated the errors by a jackknife procedure, blocking the data in groups of three configurations, and we checked that blocking in groups of different size did not produce relevant changes in the error estimates. In the first plot of Fig. 1 we show the ratio

$$
\rho(t) \equiv \frac{G_{\nabla A P}(t)}{G_{P P}(t)}
$$

as a function of $t$ for all simulated masses. Once $\rho$ has been fitted to a constant in the time interval $t_{1}-t_{2}=5-27$, a quadratic fit of the results

$$
(a \rho)=\mathcal{A}_{\rho}+\mathcal{B}_{\rho}(a m)+\mathcal{C}_{\rho}(a m)^{2}
$$

gives? (see the second plot in Fig. 1)

$$
\mathcal{A}_{\rho}=-0.00002(7) \quad \mathcal{B}_{\rho}=1.286(3) \quad \mathcal{C}_{\rho}=0.277(12)
$$

where the quoted errors are statistical only. Note that the intercept is compatible with zero. This

\footnotetext{
${ }^{1}$ Analogous correlation functions have been computed by the authors of Ref. [31]. A direct comparison with our results is not possible because we used different simulations parameters.

${ }^{2}$ From the coefficient $\mathcal{B}_{\rho}$ one can derive the value of the renormalization constant $Z_{A}$ of the "local" axial current. A detailed analysis will be presented in Ref. [26].
} 

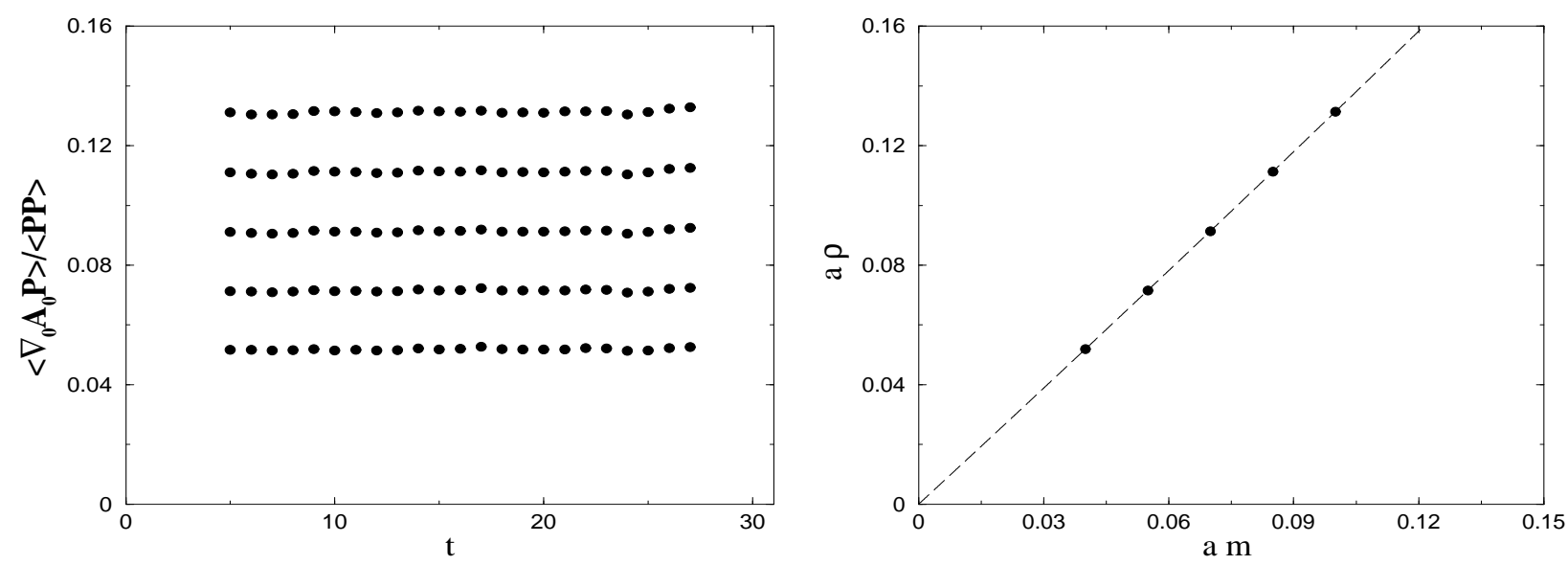

Figure 1: Left: $G_{\nabla A P} / G_{P P}$ vs. $t$ for all masses which have been simulated. Right: $(a \rho)$, obtained by fitting the data in the plot at left, as a function of the bare quark mass. The dashed line represents the result of a quadratic fit (see text).

should not come as a surprise since, even if $A_{\mu}(x)$ is not the conserved current, it has the correct behavior under global non-singlet chiral transformations.

In the quenched approximation the contribution of exact chiral zero modes of the NeubergerDirac operator is not suppressed by the fermionic determinant. In some correlation functions, this can give rise to large quenched artifacts for small masses. For example it is easy to show that $G_{P P}(t)$ receives from the unsuppressed zero modes contributions proportional to $1 / m^{2}$ and $1 / m$, which should vanish in the infinite volume limit, but can be quite sizeable for finite volume. A clever

\begin{tabular}{||c||c|c|c||c|c|c||}
\hline \hline \multicolumn{1}{||c||}{$a m$} & \multicolumn{3}{c||}{$G_{S-P}$} & \multicolumn{3}{c||}{$G_{P P}$} \\
\hline & $Z_{S-P}$ & $a M_{P}$ & $a f_{P}$ & $Z_{P P}$ & $a M_{P}$ & $a f_{P}$ \\
\hline 0.100 & $0.0040(4)$ & $0.379(6)$ & $0.089(2)$ & $0.0042(3)$ & $0.382(3)$ & $0.089(2)$ \\
0.085 & $0.0036(4)$ & $0.348(6)$ & $0.085(2)$ & $0.0039(3)$ & $0.352(4)$ & $0.085(2)$ \\
0.070 & $0.0033(4)$ & $0.315(7)$ & $0.081(2)$ & $0.0036(3)$ & $0.321(4)$ & $0.081(2)$ \\
0.055 & $0.0030(5)$ & $0.280(9)$ & $0.076(2)$ & $0.0034(3)$ & $0.287(5)$ & $0.077(2)$ \\
0.040 & $0.0026(5)$ & $0.239(11)$ & $0.071(2)$ & $0.0032(4)$ & $0.250(7)$ & $0.073(2)$ \\
\hline \hline
\end{tabular}

Table 1: Mesons masses and matrix elements for all the bare quark masses considered in the simulations, as obtained from $G_{P P}(t)$ and $G_{S-P}(t)$.

way to avoid such artifacts has been proposed by the authors of Ref. [32], who noticed that the zero modes, because of their chirality properties, contribute equally to the $G_{P P}$ and $G_{S S}$ correlation 

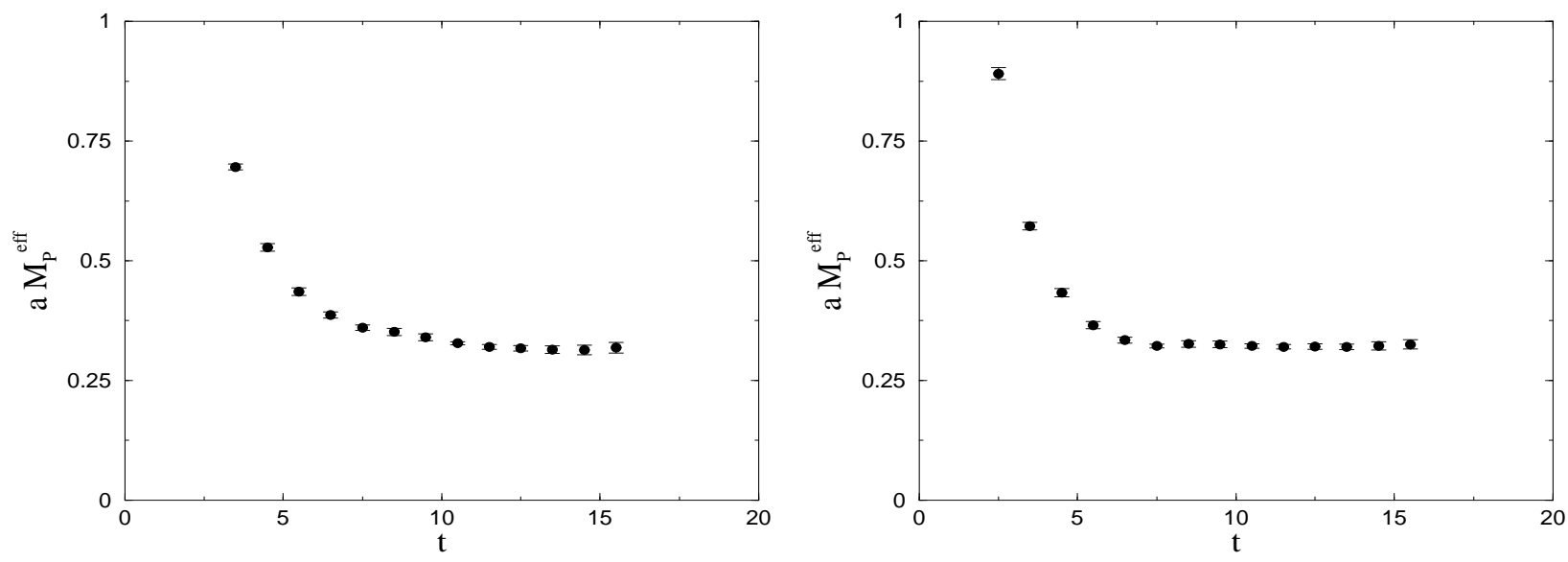

Figure 2: Left: A representative example $(a m=0.070)$ of the effective mass from $G_{S-P}(t)$. Right: as at left but from $G_{P P}(t)$.

functions, so that their contributions cancel in the difference

$$
G_{S-P}(t)=G_{P P}(t)-G_{S S}(t)
$$

which can also be used to extract the pseudoscalar meson mass and decay constant, since the contributions from the heavier scalar mesons fall off faster. The drawback is, of course, that the plateau in the effective mass and, correspondingly, the range that can be used for the cosh fit become shorter. Nevertheless we found the plateau in the $G_{S-P}$ to be long enough to permit a meaningful fit. We fitted $G_{S-P}(t)$ to a single particle propagator with a cosh dependence on $t$,

$$
G_{S-P}(t)=\frac{Z_{S-P}}{a M_{P}} \exp \left(-\frac{1}{2} a M_{P} T\right) \cosh \left(a M_{P}\left(\frac{T}{2}-t\right)\right)
$$

in the time interval $t_{1}-t_{2}=12-16$. The lower limit was fixed at the point where we found stabilization of the effective meson masses. The results of the fits are also given in Table 11 and an example of the effective meson mass from $G_{S-P}(t)$ is shown in first plot of Fig. 2. We also performed a two cosh fit of $G_{S-P}(t)$ finding consistent results.

We have also fitted the correlation functions $G_{P P}(t)$ to a single particle propagator with a cosh dependence on $t$, as in Eq. (34), in the time interval $t_{1}-t_{2}=10-16$. As before the lower limit is fixed as the point at which the values of the effective meson masses become stable. We report our results in Table 1 and in the second plot of Fig. 2 we give an example of the effective meson mass as extracted from $G_{P P}(t)$.

We illustrate in Fig. 3 the values for $\left(a M_{P}\right)^{2}$, obtained from $G_{S-P}(t)$ and $G_{P P}(t)$, as a function of the bare quark mass am. In both cases a linear behavior

$$
\left(a M_{P}\right)^{2}=\mathcal{A}_{M_{P}}+\mathcal{B}_{M_{P}}(a m)
$$



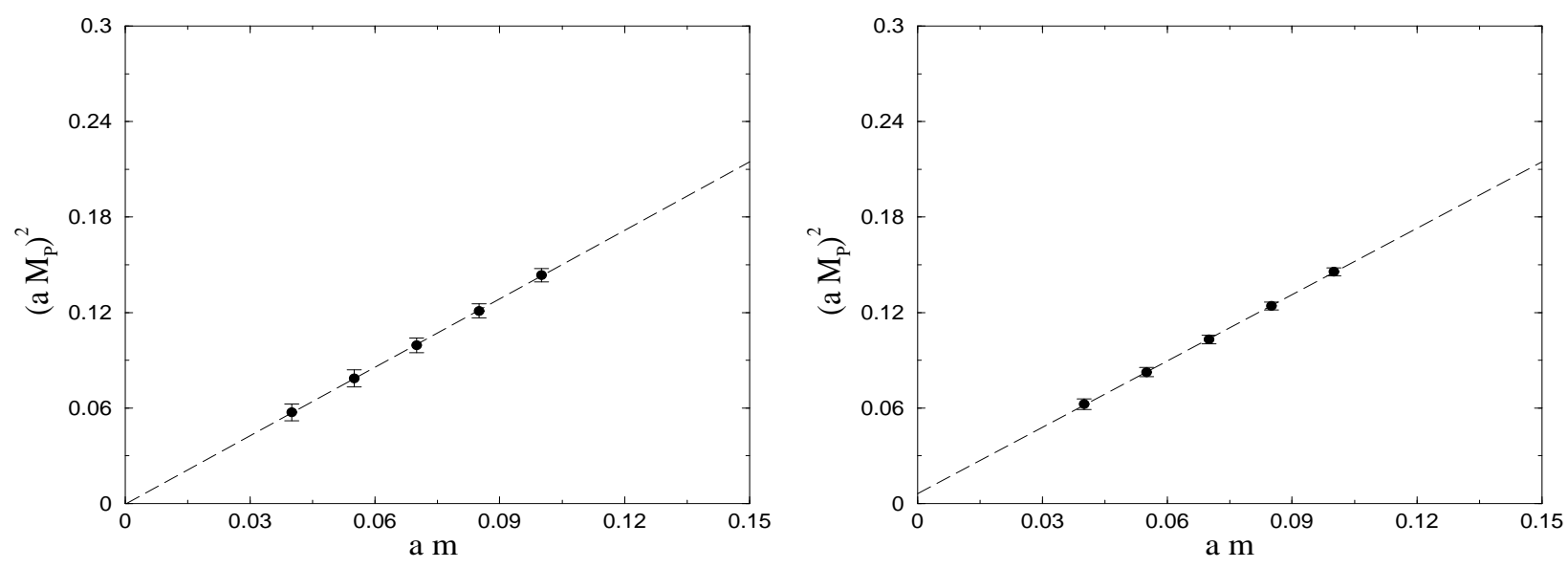

Figure 3: $\left(a M_{P}\right)^{2}$ vs. $a m$ as obtained from $G_{S-P}(t)$ (left) and $G_{P P}(t)$ (right). The dashed lines represent the results of a linear fit.

fits very well the data with

$$
\mathcal{A}_{M_{P}}=-0.0005(68) \quad \mathcal{B}_{M_{P}}=1.43(7)
$$

for the masses obtained from $G_{S-P}(t)$ and

$$
\mathcal{A}_{M_{P}}=0.006(4) \quad \mathcal{B}_{M_{P}}=1.39(3)
$$

for those obtained from $G_{P P}(t)$. We see that the parameters of the two fits are compatible and that both intercepts vanish within statistical errors. In particular, one does not notice any sign of the singular contributions from zero modes in the masses obtained from the pseudoscalar correlation functions. These are expected to show up at some point, but one would probably need much higher statistical accuracy and lower values of $m$ to bring them into evidence.

Contrary to the pseudoscalar masses, the results for the matrix elements, parameterized as in Eq. (34) by the factors $Z_{S-P}$ and $Z_{P P}$ for $G_{S-P}(t)$ and $G_{P P}(t)$, respectively, show more significant differences. In Fig. 1 we reproduce the results we obtained for $Z_{S-P}$ and $Z_{P P}$ respectively. We see that, while $Z_{S-P}$ exhibits a linear behavior as function of $a m$, the graph for $Z_{P P}$ shows a clear indication of curvature. Linear fits of the form

$$
Z_{i}=\mathcal{A}_{i}+\mathcal{B}_{i}(a m)
$$

give

$$
\begin{aligned}
\mathcal{A}_{S-P} & =0.0016(7) & & \mathcal{B}_{S-P}=0.024(6) \\
\mathcal{A}_{P P} & =0.0025(4) & & \mathcal{B}_{P P}=0.016(3)
\end{aligned}
$$



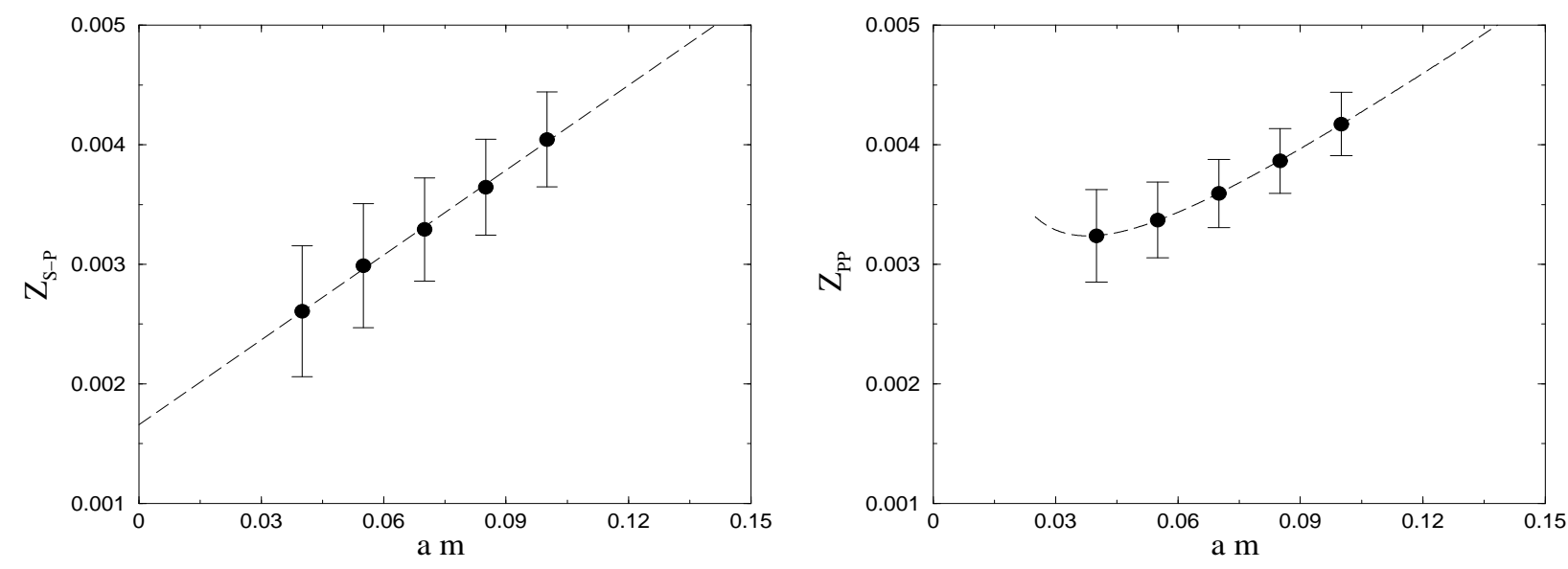

Figure 4: Left: $Z_{S-P}$ vs. $(a m)$; the dashed line represents the result of a linear fit. Right: $Z_{P P}$ vs. $(a m)$; the dashed line represents a fit of the form of Eq. (41).

Within the quite large statistical errors, the intercept and the slope obtained from $G_{S-P}(t)$ are still compatible with those derived from $G_{P P}(t)$. However the central values are quite different. If we interpret the curvature in the graph of $Z_{P P}$ as due to pole terms from the zero modes and try a simple fit of the form

$$
Z_{P P}=\mathcal{A}_{P P}+\mathcal{B}_{P P}(a m)+\frac{\mathcal{D}_{P P}}{a m}
$$

we obtain

$$
\mathcal{A}_{P P}=0.0014(3) \quad \mathcal{B}_{P P}=0.024(2) \quad \mathcal{D}_{P P}=0.000035(16)
$$

Now the $\chi^{2} /$ d.o.f of the fit turns out to be much smaller and the central values of intercepts and slope parameters for the fits of $Z_{S-P}$ and $Z_{P P}$ are much closer. The fact that the curvature shows up only in the results for $Z_{P P}$ points to the fact that what we are seeing is the effect of the unsuppressed zero modes, and not of chiral logarithms which would affect both sets of results (and would most likely become noticeable at much smaller values of $a m$ ). On account of the above, we will use $G_{S-P}(t)$ to derive our further results. It must also be said that some of the observables will be calculated directly at $m \simeq m_{s} / 2$ (see below), and for these observables the difference between $Z_{P P}$ and $Z_{S-P}$ is irrelevant within our statistical error.

The pseudoscalar decay constant is defined through Eq. (22) and we calculated its value directly from the parameters extracted from a cosh fit to $G_{S-P}(t)$

$$
a f_{P}=2(a m) \frac{\sqrt{Z_{S-P}}}{\left(a M_{P}\right)^{2}}
$$

The values we obtained for $a f_{P}$ in correspondence to each simulated mass are reproduced in Table 1 (where we also reproduce the values derived from $G_{P P}(t)$ ) and are shown in Fig. 5. The dashed and 


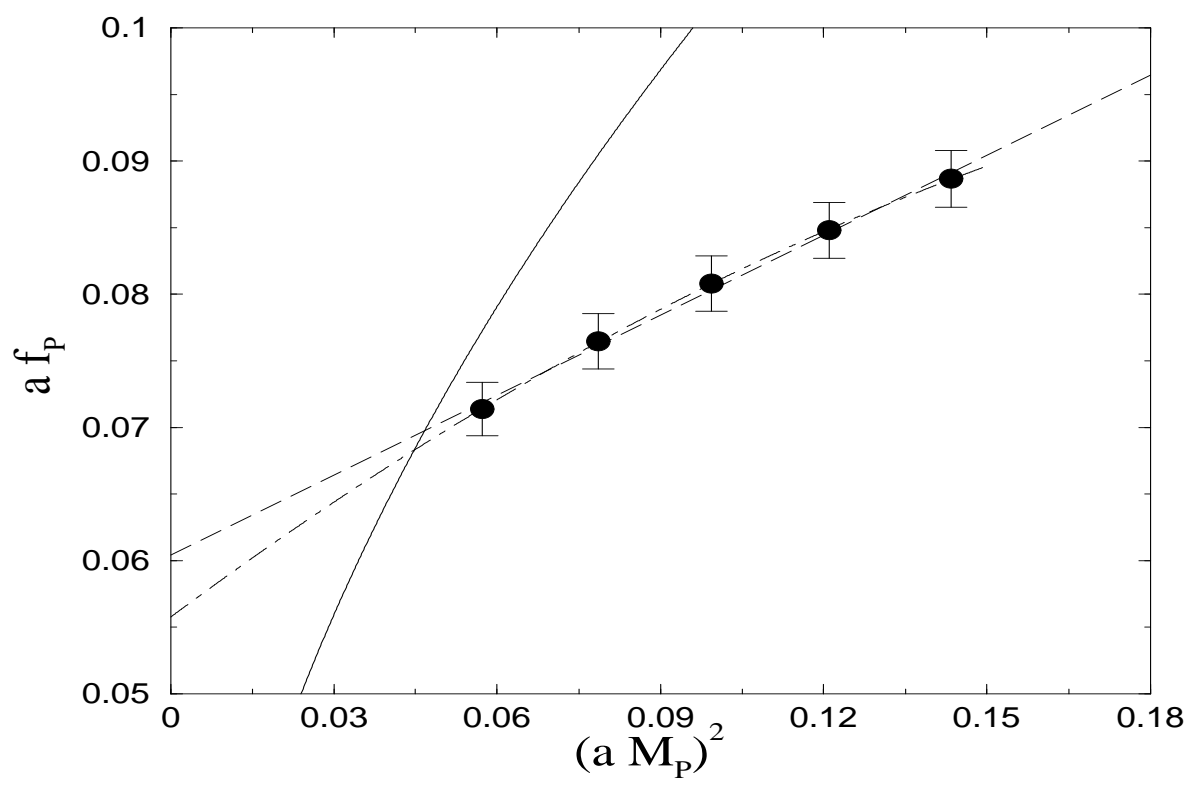

Figure 5: The procedure used to derive $a f_{K}$ and $a M_{K}$. The dashed and dashed-dotted lines represent fits to our numerical results. The solid line represents the curve $\left(a f_{P}\right)=C_{s l}\left(a M_{P}\right)$, where the coefficient $C_{s l}=f_{K}^{e x p} / M_{K}^{e x p}$.

the dot-dashed lines show the results of a linear and a quadratic fit in $\left(a M_{P}\right)^{2}$, respectively. The data slightly favors a quadratic fit, but our statistical accuracy is insufficient to rule out a linear fit as inconsistent with the data. It is interesting to observe, though, that we obtain $f_{K} / f_{\pi} \simeq 1.14$ and $\simeq 1.23$ from the linear and quadratic fits, respectively, with the results from the quadratic fit in much better agreement with the experimental value.

Starting from our values for $M_{P}$ and $f_{P}$, we fix the lattice spacing $a^{-1}$ and the physical meson masses by using the method of "lattice physical planes" [5]. This avoids recourse to a chiral extrapolation for observables where it is not really needed. In the plane $\left[a f_{P},\left(a M_{P}\right)^{2}\right]$, we plot our lattice data as well as the curve $\left(a f_{P}\right)=C_{s l}\left(a M_{P}\right)$ (the solid line in Fig. 每), where the coefficient of proportionality $C_{s l}$ (sl for strange-light) is fixed by the experimental value for the ratio $f_{K}^{\exp } / M_{K}^{\exp }$. (1) The point where the two lines meet determines $a f_{K}$ as well as $a M_{K}$. Since our statistical accuracy does not allow us to discriminate between a linear and quadratic fit to the data for $a f_{P}$ vs. $\left(a M_{P}\right)^{2}$ , we just use the linear fit for the tiny extrapolation to the "Kaon" point (see Fig. 5). Our results are

$$
a M_{K}=0.216(8) \quad a f_{K}=0.0698(26)
$$

(A quadratic fit produces a result which differs from the above by far less than the statistical

\footnotetext{
${ }^{3}$ Throughout the paper we use the following experimental numbers: $M_{K}^{\text {exp }}=495 \mathrm{MeV}, f_{K}^{e x p}=160 \mathrm{MeV}$. We neglect the experimental errors which are well below our statistical and systematic errors.
} 
errors). To set the lattice spacing we compare the value of $a f_{K}$ with its experimental value and we get

$$
a_{f_{K}}^{-1}=2.29(9)
$$

This is the value of the lattice spacing we will use throughout the paper. Again we stress that up to this stage we did not have to perform any chiral extrapolation, but derived all physical information staying close to the region of quark masses used in the actual calculations.

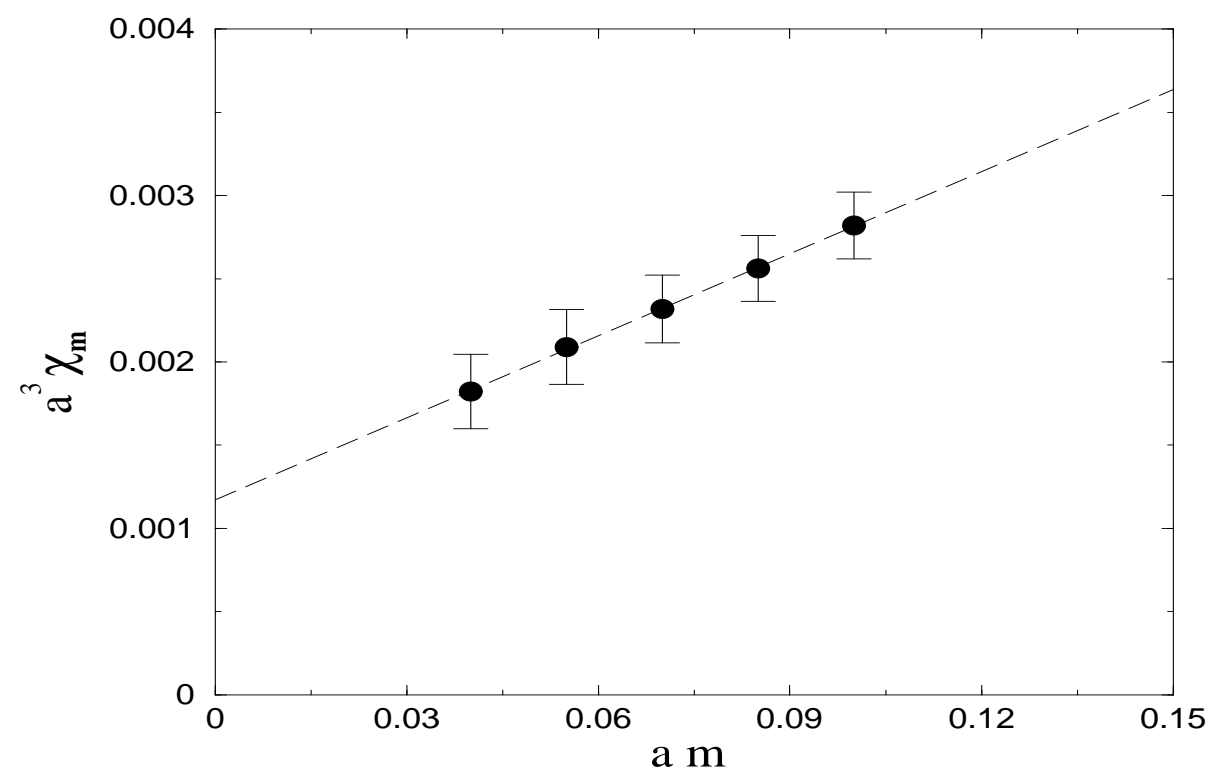

Figure 6: The data used to derive the chiral condensate from the GMOR relation.

From our data, we can also compute the chiral condensate by using the GMOR relation of Eq. (21). In Fig. 6 we show the quantity

$$
a^{3} \chi_{m}=-(a m) \frac{Z_{S-P}}{\left(a M_{P}\right)^{2}}
$$

as a function of the bare quark mass. The data exhibits a very good linear behavior and a linear fit leads to

$$
a^{3} \chi=-0.00117(27)
$$

We performed also a quadratic fit to the data, obtaining a result consistent with zero for the coefficient of the quadratic term, but with an error twice as large in the intercept, because of the additional degree of freedom. Therefore we conclude that we see no indication for a quadratic term in the fit within our statistical error.

The chiral condensate can also be computed directly from Eq. (16). However, away from the chiral limit, the r.h.s. of that equation contains power divergent terms (in $a^{-1}$, see Eq. (20)) and, in 
the quenched approximation, infrared divergent contributions (in $m$ ) due to the unsuppressed zero modes. Therefore quite a severe extrapolation from our simulated data is needed to determine its chiral value. One can take advantage, though, of a computational strategy similar to that used in Eq. (33). To remove the contribution due to unsuppressed zero modes, we subtract the scalar-scalar correlation function from the r.h.s of Eq. (17). From a quadratic fit in the quark mass we obtain

$$
a^{3} \chi(a)=-0.00117(42)
$$

which is in remarkable agreement with Eq. (47). Of course, the larger error is an indication of the difficulty of the extrapolation. Larger statistics and a careful treatment of the zero mode contributions would be needed to obtain more reliable and precise results by the direct method.

\section{Physical Results}

In this section we will use our lattice results to infer the renormalized values of the sum of the strange and average up-down quark masses and of the chiral condensate. To obtain our final values, we still need the value of the the scalar renormalization constant $Z_{S}(\mu a)$, which we have computed non-perturbatively in the RI/MOM scheme following the approach proposed in [17]. The details of these calculations will be presented in a separate paper [26]. The result we obtained in the RI scheme is

$$
Z_{S}^{\mathrm{RI}}(2 \mathrm{GeV})=1.24(5)
$$

where the error is mainly systematics due to the chiral extrapolation and to the uncertainty in the value of the renormalization scalef. Judging from the comparison of the data at different renormalization scales with the logarithmic evolution predicted by the renormalization group equations at $\mathrm{N}^{2} \mathrm{LO}$, the discretization errors appear to be well below the error in Eq. (49). The result in Eq. (49) differs by about 10\% from the bare perturbation theory determination at one loop $\left[Z_{S}^{\mathrm{RI}}(2 \mathrm{GeV})\right]^{P T}=1.11[23,24]$. However if we use $\alpha_{s}^{\overline{\mathrm{MS}}}$ as expansion parameter we obtain a result consistent within errors with Eq. (49). By using $\mathrm{N}^{2} \mathrm{LO}$ continuum perturbation theory [33] with $N_{f}=0$ and $\Lambda_{Q C D}=0.238(19)$ 11] to convert the result in Eq. (49) into the $\overline{\mathrm{MS}}$ scheme, we obtain

$$
Z_{S}^{\overline{\mathrm{MS}}}(2 \mathrm{GeV})=1.41(6)
$$

Had we used the experimental $\mathrm{N}^{2} \mathrm{LO}$ results from $\alpha_{s}\left(M_{Z}\right)=0.118$, we would have obtained a value of the scalar renormalization constant $\sim 10 \%$ higher than the one given above. The difference can be taken as an indication of the systematic error introduced by the quenched approximation. Therefore we will add this uncertainty in quadrature to our final estimate of the systematic error. $\mathrm{N}^{3} \mathrm{LO}$ perturbative computations are available for the RI - $\overline{\mathrm{MS}}$ matching of the scalar renormalization constant [34], but the difference with the $\mathrm{N}^{2} \mathrm{LO}$ results is much below the error induced by the quenching ambiguity discussed above. If we had used the procedure proposed recently in Ref. 27] on our data, we would have obtained a value for $Z_{S}^{\overline{\mathrm{MS}}}(2 \mathrm{GeV})$ which agrees within the errors with the one in Eq. (50), but with a central value $\simeq 10 \%$ higher.

\footnotetext{
${ }^{4}$ We stress again that this number is obtained at $\beta=6.0$ for $\rho=1.4$ and $r=1.0$.
} 
We finally combine this last result with the numbers presented in the previous section to obtain our main physical results. From the value of $a M_{K}$ in Eq. (44) and from the lattice spacing in Eq. (45), we obtain for the combination of bare quark masses

$$
m_{s}(a)+\hat{m}(a)=149(9) \mathrm{MeV}
$$

where the error is only statistical. Since our volume is fairly large, we expect our main sources of systematic errors to come from discretization effects and from the quenched approximation. For a rough estimate of the systematic error due to quenching approximation we can use the results in Ref. [36]. From these one sees that, within the quenched approximation, the use of different observables to calculate the lattice spacing can produce differences in the results of $\sim 10 \%$. Had we used $r_{0}$ [37] to fix our lattice spacing we would have obtained a number $\sim 7 \%$ higher than the one in Eq. (51). Combining this fact with the results in [12], we would infer that discretization uncertainties are below $10 \%$. In order to be conservative, we will take $15 \%$ as the estimate of our overall systematic error in the renormalized quark masses due to quenching and discretization effects. A more precise estimate of the systematic errors will need much more extensive simulations, which at present would be beyond our capability and the exploratory scope of this work. Combining the results in Eqs. (50) and (51) we obtain

$$
\left(m_{s}+\hat{m}\right)^{\mathrm{RI}}(2 \mathrm{GeV})=120 \pm 7 \pm 21 \mathrm{MeV}
$$

which represents one of the major results of this paper. By using Eqs. (11) and (50) the above translates to

$$
m_{s}^{\overline{\mathrm{MS}}}(2 \mathrm{GeV})=102 \pm 6 \pm 18 \mathrm{MeV}
$$

This result agrees very well with the current lattice world average [16].

Insofar as the value of the condensate is concerned, if we used the standard two-step approach, i.e. first measure the dimensionless condensate, see Eqs. (47) or (48), and then multiply it by the cubic power of the lattice spacing, the result would be affected by a very large systematic error due to the uncertainty in the determination of the lattice spacing in quenched simulations. Instead, we will use an alternative method [6]. We write the GMOR relation (23) for the renormalized condensate as follows

$$
\chi(a)=-\frac{1}{4} f_{\chi}^{2} \mathcal{B}_{M_{P}} a^{-1}
$$

where $\mathcal{B}_{M_{P}}$ is defined in Eq. (36) and $f_{\chi}=0.1282 \mathrm{GeV}$ is the "experimental" value, in physical units, of the pseudoscalar decay constant extrapolated to the chiral limit. While computing the condensate from the above formula relies on an additional element of experimental information, it has several advantages. The most important is that, by expressing the condensate in terms of $f_{\chi}$, we are left with only one power of the UV cutoff $a^{-1}$. Another advantage is that, if we assume that the relation between $M_{P}$ and $m$ stays substantially linear for a range of quark masses extending to $\sim m_{s}$ (and our numerical results validate this, see Fig. 3), then there is no need for an extrapolation to the chiral limit to evaluate $\mathcal{B}_{M_{P}}$. With this method we obtain

$$
\langle\bar{\psi} \psi\rangle^{\mathrm{RI}}(2 \mathrm{GeV})=-0.0167 \pm 0.0010 \pm 0.0029 \mathrm{GeV}^{3}
$$


where the estimate of the systematic error has been made using the same criteria we used for estimate of the error in the quark mass. This is our best value for the chiral condensate. It is interesting to note that, if we had used the standard technique, starting from Eq. (47) and with the lattice spacing of Eq. (45), we would have obtained a result with a central value very close to the value in Eq. (55), but with a much higher systematic error.

Finally, from Eq. (55) and $\mathrm{N}^{2} \mathrm{LO}$ matching, we get

$$
\langle\bar{\psi} \psi\rangle^{\overline{\mathrm{MS}}}(2 \mathrm{GeV})=-0.0190 \pm 0.0011 \pm 0.0033 \mathrm{GeV}^{3}=-[267 \pm 5 \pm 15 \mathrm{MeV}]^{3}
$$

This result is in very good agreement with the result obtained by the authors of Refs. [35, 27], while it is smaller than the result in Ref. [38], even if still compatible within errors. Our result is also compatible within errors with the number obtained few years ago in Ref. [6] with Wilson-type fermions. We expect, though, the systematics due to the discretization effects to be smaller $\left(\mathcal{O}\left(a^{2}\right)\right)$ in the result reported in Eq. (56) than the error $(\mathcal{O}(a))$ which affects the determination in Ref. [6].

\section{Conclusions}

In the overlap regularization chiral symmetry is preserved at finite lattice spacing and finite volume, therefore there is no mixing among operators of different chirality. As a consequence no additive quark mass renormalization is required and no fine tuned parameters are needed to compute $\mathcal{O}(a)$ improved masses and matrix elements. Our results have indeed produced a remarkable verification of "good chiral behavior" both in the axial Ward identity and in the pseudoscalar masses.

In this paper we presented the results of the first computation of $\left(m_{s}+\hat{m}\right)$ with overlap fermions in the quenched approximation. To avoid uncertainties due to lattice perturbation theory, we computed the multiplicative renormalization constant $Z_{S}(\mu a)$ non-perturbatively in the RI/MOM scheme. Our main results have been summarized in the introduction. While the systematics errors due to quenching are common to previous calculations, the other systematic errors (mostly discretization effects) are different than with other lattice regularizations and likely to be smaller, because of chiral symmetry. We also computed the chiral condensate $\langle\bar{\psi} \psi\rangle$ from the GMOR relation and directly. Even if, given our statistical and systematic errors, the former method is more reliable, it is rewarding to notice that the two determinations are in good agreement.

The calculation of light quark masses uses many of the ingredients needed for a lattice calculation of weak matrix elements, although the latter is computationally more demanding. From this point of view, the very good agreement between our results for the quark masses and the current lattice world average bodes well for the use of the overlap formalism also for matrix element calculations. Our investigation has been mostly of exploratory nature. One would need to extend it to larger volumes and better statistics. One should also find a more direct way to isolate and account for the effects of the zero modes. Nevertheless, we believe that it gave a strong indication that the overlap formalism can be used effectively, with known algorithms and the present generation of computers, for large scale QCD calculations, at least in the quenched approximation. Thus we would conclude that it represents a very promising non-perturbative regularization for solving long 
standing problems, such as the proof of the $\Delta I=1 / 2$ rule and the calculation of $\epsilon^{\prime} / \epsilon$, which would be hard to address with conventional regularizations.

\section{Acknowledgments}

We wish to thank Boston University's Center for Computational Science and Office of Information Technology at Boston University for generous allocations of supercomputer time and the Scientific Computing and Visualization group for invaluable technical assistance. We also gratefully acknowledge the use of the gauge configurations produced by the authors in Ref. [28]. This work has been supported in part under DOE grant DE-FG02-91ER40676.

\section{References}

[1] J. Gasser, H. Leutwyler, Phys. Rep. 87 (1982) 77.

[2] H. Leutwyler, Phys. Lett. B 378 (1996) 313.

[3] C. R. Allton et al., Nucl. Phys. B 431 (1994) 667.

[4] R. Gupta, T. Bhattacharya, Phys. Rev. D 55 (1997) 7203.

[5] C. R. Allton, V. Gimenez, L. Giusti, F. Rapuano, Nucl. Phys. B 489 (1997) 427.

[6] V. Gimenez, L. Giusti, F. Rapuano, M. Talevi, Nucl. Phys. B 540 (1999) 472;

V. Gimenez, L. Giusti, F. Rapuano, M. Talevi, Nucl. Phys. B 531 (1998) 429;

L. Giusti, F. Rapuano, M. Talevi, A. Vladikas, Nucl. Phys. B 538 (1999) 249.

[7] N. Eicker et al. [SESAM collaboration], Phys. Rev. D 59 (1999) 014509.

[8] D. Becirevic et al., Phys. Lett. B 444 (1998) 401.

[9] S. Aoki et al. [JLQCD Collaboration], Phys. Rev. Lett. 82 (1999) 4392.

[10] T. Blum, A. Soni, M. Wingate, Phys. Rev. D 60 (1999) 114507.

[11] S. Capitani, M. Luscher, R. Sommer, H. Wittig, Nucl. Phys. B 544 (1999) 669.

[12] J. Garden, J. Heitger, R. Sommer, H. Wittig [ALPHA Collaboration], Nucl. Phys. B 571 (2000) 237.

[13] A. Ali Khan et al. [CP-PACS Collaboration], Phys. Rev. Lett. 85 (2000) 4674.

[14] S. Narison, Nucl. Phys. (Proc. Suppl.) 86 (2000) 242 and references therein.

[15] M. Davier et al., Nucl. Phys. (Proc. Suppl.) 98 (2001) 319 and references therein. 
[16] V. Lubicz, Nucl. Phys. (Proc. Suppl.) 94 (2001) 116.

[17] G. Martinelli, C. Pittori, C.T. Sachrajda, M. Testa, A. Vladikas, Nucl. Phys. B 445 (1995) 81.

[18] R. Narayanan, H. Neuberger, Phys. Lett. B 302 (1993) 62;

R. Narayanan, H. Neuberger, Nucl. Phys. B443 (1995) 305.

H. Neuberger, Phys. Lett. B 417 (1998) 141;

H. Neuberger, Phys. Lett. B 427 (1998) 353.

[19] P. H. Ginsparg, K. G. Wilson, Phys. Rev. D 25 (1982) 2649.

[20] M. Lüscher, Phys. Lett. B 428 (1998) 342.

[21] P. Hasenfratz, V. Laliena, F. Niedermeyer, Phys. Lett. B 427 (1998) 125.

[22] P. Hasenfratz, Nucl. Phys. B 525 (1998) 401.

[23] C. Alexandrou, H. Panagopoulos, E. Vicari, Nucl. Phys. B 571 (2000) 257.

C. Alexandrou, E. Follana, H. Panagopoulos, E. Vicari, Nucl. Phys. B 580 (2000) 394.

[24] S. Capitani, L. Giusti, Phys. Rev. D 62 (2000) 114506;

S. Capitani, L. Giusti, Phys. Rev. D 64 (2001) 014506.

[25] K. Jansen et al., Phys. Lett. B 372 (1996) 275;

M. Lüscher, S. Sint, R. Sommer, H. Wittig, Nucl. Phys. B 491 (1997) 344.

[26] L. Giusti, C. Hoelbling, C. Rebbi, in preparation.

[27] P. Hernández, K. Jansen, L. Lellouch, H. Wittig, hep-lat/0106011.

[28] We retrieved the first 54 gauge configurations OSU_Q60a produced by G. Kilcup, D. Pekurovsky, L. Venkataraman, Nucl. Phys. (Proc. Suppl.) 53 (1997) 345 from the repository at the "Gauge Connection" (http://qcd.nersc.gov/).

[29] H. Neuberger, Phys. Rev. Lett. 81 (1998) 4060;

R. G. Edwards, U. M. Heller, R. Narayanan, Nucl. Phys. B 540 (1999) 457.

[30] R. G. Edwards, U. M. Heller, R. Narayanan, Phys. Rev. D 59 (1999) 094510.

[31] S. J. Dong, F. X. Lee, K. F. Liu, J. B. Zhang, Phys. Rev. Lett. 85 (2000) 5051.

[32] T. Blum et al., hep-lat/0007038 and hep-lat/0007038.

[33] T. van Ritbergen, J.A.M. Vermaseren and S.A. Larin, Phys. Lett. B400 (1997) 379;

J.A.M. Vermaseren, S.A. Larin and T. van Ritbergen, Phys. Lett. B405 (1997) 327;

K.G. Chetyrkin, Phys. Lett. B404 (1997) 161;

E. Franco and V. Lubicz, Nucl. Phys. B531 (1998) 641.

[34] K. G. Chetyrkin, A. Retey, Nucl. Phys. B 583 (2000) 3. 
[35] P. Hernandez, K. Jansen, L. Lellouch, Phys. Lett. B 469 (1999) 198;

[36] S. Aoki et al. [CP-PACS-Collaboration], Phys. Rev. D 60 (1999) 114508.

[37] M. Guagnelli, R. Sommer, H. Wittig [ALPHA collaboration], Nucl. Phys. B 535 (1998) 389.

[38] T. DeGrand [MILC collaboration], Phys. Rev. D 63 (2001) 034503;

T. DeGrand [MILC Collaboration], hep-lat/0107014. 